\title{
Adapting Distance Based Clustering Concept to a Heterogeneous Network
}

\author{
N. Laloo, M. Z. A. A. Aungnoo, and M. S. Sunhaloo
}

\begin{abstract}
We propose an improved energy consumption model for a heterogeneous network by adapting an existing distance based clustering concept to it. We assume that if the distance based clustering concept has improved a homogeneous network, the same concept if applied to a heterogeneous network will improve the latter. A heterogeneous network, which is an improved version of a homogeneous network, is considered. We apply our model on the Stable Election Protocol, which is a common routing algorithm designed for heterogeneous networks. We show that the Stable Election Protocol is improved in terms of longer lifetime at the expense of the stability period. Finally, we conclude by explaining our results and suggest improvement for the stability period.
\end{abstract}

Index Terms-Distance-based clustering, heterogeneous network, stable election protocol, wireless sensor network.

\section{INTRODUCTION}

With advancement in computing and communication, wireless sensor network (WSN) is gaining more and more interest in different areas of research [1]-[4]. A wireless sensor network is composed of a sink and numerous wireless sensor nodes, which together make up the wireless sensor field [5]. These nodes are capable of sensing, processing and communication. The nodes may operate in inaccessible region. However, the network remains useful as long as there are alive sensor nodes. Nodes in the network are often deployed randomly in unattended region [1]. The deployed nodes may all be similar with same energy level resulting in a homogeneous network or in cases where the nodes have varying energy level a heterogeneous network is formed. Much emphasis is being placed at prolonging the network lifetime.

In this paper an existing model of the energy consumption of a heterogeneous network is considered. We study concepts that improve the energy consumption in a homogeneous network. We then improve the heterogeneous network by applying one of the concepts of homogeneous network to the heterogeneous network using distance-based clustering. The new concept is then applied on the Stable Election Protocol (SEP) [6].

We found that the SEP algorithm is improved in terms of lifetime though the first node death occurs earlier than in the

Manuscript received April 7, 2014; revised May 20, 2014.

$\mathrm{N}$. Laloo is with the Department of Industrial Systems Engineering, School of Innovative Technologies and Engineering, University of Technology, Mauritius (e-mail: n.laloo@umail.utm.ac.mu).

M. Z. A. A. Aungnoo was with the Department of Industrial Systems Engineering, School of Innovative Technologies and Engineering, University of Technology, Mauritius (e-mail: z_furyan@ @otmail.com).

M. S. Sunhaloo is with the School of Innovative Technologies and Engineering, University of Technology, Mauritius (e-mail: ssunhaloo@umail.utm.ac.mu). original SEP.

The remaining part of the paper is organized as follows: in Section II we explain a common model for energy consumption in homogeneous and heterogeneous networks. The Low Energy Adaptive Cluster Hierarchy Routing Protocol (LEACH) [7] and SEP are described in Section III. In Section IV a brief overview of the energy enhancement concepts for homogeneous network is given followed by a description of the distance-based concept as applied to the distance based clustering algorithm in Section V. In section 6, we explain how the distance-based concept of EECS algorithm may be applied to SEP to improve a heterogeneous network, which to the best of our knowledge has not been done before. Simulation results are presented in Section VII and finally in Section VIII we explain our results and conclude.

\section{ENERGY MODELING IN HOMOGENEOUS AND HETEROGENEOUS WIRELESS SENSOR NETWORK}

Most of the work that improve on the energy consumption of wireless sensor networks focus mainly on homogeneous networks, where all the sensors are similar in terms of energy and hardware complexity. Heterogeneous networks arise from having multiple sensor types with different energy levels and complexity within the same network and form the basis of this paper.

We consider a simple heterogeneous network where the sensors are equipped with varying battery power. The particular network model considered is to have sensors randomly dropped from a helicopter on a remote field. The sensors are responsible for sensing the data, processed the sensed data partly and forward the result to a remote base station (BS) for further processing. The main issue we are interested in is how to improve the lifetime of such a sensor network for a given amount of energy. The lifetime of a sensor network is considered to be the period before all the sensor nodes die. Thus in prolonging the lifetime of a sensor network, we attempt to balance the power exhaustion from one sensor to another.

Data from the sensing nodes may be collected in different ways namely direct transmission whereby the sensor directly sends sensed data to the base station independently of each other nodes [7]. This method though does not require any communication between the sensors is inefficient as the network size increases. Another approach is though multi hop based on random traffic pattern [8]-[10]. A third approach, which is as well the focus of this paper, is clustering approach where the sensors form clusters dynamically with surrounding sensors [7]. The sensors organize themselves such that one sensor is elected as the cluster head for each round. The node selected as cluster head is responsible for 
collecting, aggregating the data, and consequently forwarding the data to the BS.

The typical model for energy consumption in homogeneous and heterogeneous networks is as shown in Fig. 1 below:

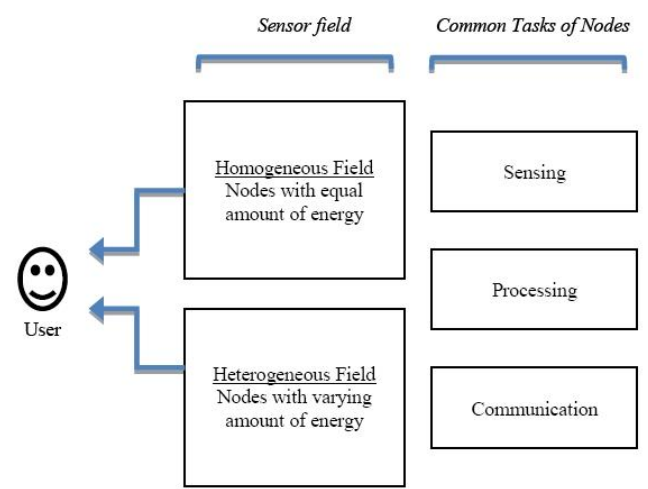

Fig. 1. Tasks of nodes in homogeneous and heterogeneous WSN fields.

Fig. 1 illustrates the tasks of nodes in both the homogeneous and heterogeneous networks. Though the starting energy level of the nodes in the different fields may vary, the basic tasks of the nodes remain practically the same. Thus, a concept, which improves a homogeneous network, should theoretically improve a heterogeneous network as well.

\section{LEACH AND SEP}

The Low Energy Adaptive Clustering Hierarchy protocol presented by Heinzelman, Chandrakasan \& Balakrishnan [7] is a cluster-based application specific protocol whereby the nodes organized themselves into local clusters with one node acting as the cluster head $(\mathrm{CH})$ and the other nodes acting as normal nodes [1]. LEACH assumes homogeneity of the nodes such that the nodes have equal energy and processing power. LEACH ensures prolonged network lifetime and minimum energy usage by rotating the $\mathrm{CH}$ nodes and allowing data fusion within the clusters.

LEACH operates in rounds with each round being divided into the setup and the steady phases. Clusters are formed in the setup phase and data transmission occurs in the steady phase whereby nodes send data to the $\mathrm{CH}$ nodes, which in turn forward the data to the BS An otimal number of cluster heads, is guaranteed and probabilistic methods are applied to determie the CHs based on (1),

$$
T_{n}(t)= \begin{cases}\frac{p}{1-p\left(r \bmod \frac{1}{p}\right)}, & n \in G, \\ 0 & , \text { otherwise },\end{cases}
$$

where $T_{n}$ is the threshold, $p$ is the percentage of $\mathrm{CH}, r$ is the current round, and $G$ the set of non $\mathrm{CH}$ in the last $1 / p$ rounds.

SEP is a common heterogeneous algorithm which is based on LEACH and which improves upon LEACH by considering extra energy nodes [6]. SEP introduces heterogeneity in terms of higher energy level in certain nodes. Furthermore, SEP uses nodes energy and an additional parameter compared to $\mathrm{LEACH}$ to elect its $\mathrm{CH}$. The percentage of advanced nodes and the percentage of additional energy in the advanced nodes are considered. In
SEP both the normal nodes and the advanced nodes may be elected as a $\mathrm{CH}$ node, thus giving rise to two threshold values. If the optimal probability for normal nodes to become a $\mathrm{CH}$ is $P_{n r m}$ and the optimal probability for advanced nodes to become a CH is $P_{a d v}$, then (2) and (3) are used to determine the $\mathrm{CH}$ in SEP.

$$
\begin{gathered}
T\left(S_{n r m}\right)=\left\{\begin{array}{cl}
\frac{p_{n r m}}{1-p_{n r m}\left(r \bmod \frac{1}{p_{n r m}}\right)}, & S_{n r m} \in G_{n r m}, \\
0 & \text { otherwise }
\end{array}\right. \\
T\left(S_{a d v}\right)=\left\{\begin{array}{cl}
\frac{p_{a d v}}{1-p_{a d v}\left(r \bmod \frac{1}{p_{a d v}}\right)}, & S_{a d v} \in G_{a d v}, \\
0 & \text { otherwise },
\end{array}\right.
\end{gathered}
$$

where $r$ is the current round, $G_{n r m}$ is the set of normal nodes which have not been elected as $\mathrm{CH}$ within the last $1 / p_{\text {nrm }}$ rounds and $G_{a d v}$ is the set of normal nodes which have not been elected as $\mathrm{CH}$ within the last $1 / p_{a d v}$ rounds.

The threshold values of SEP consider the heterogeneous parameters $m$ and $\alpha$, and consequently extract optimal number of clusters thus saving energy. $m$ is the percentage of advanced nodes and $\alpha$ is the percentage of additional energy in advanced nodes.

\section{ENERGY ENHANCEMENT CONCEPTS IN HOMOGENEOUS NETWORKS}

Different concepts exist to enhance energy in WSN [11]. Topology control is use to ensure finding the optimal subset of nodes to maintain connectivity and alleviating network redundancy. Data driven approaches including data reduction schemes, data acquisition schemes and data aggregation are considered to address the problem of unneeded samples and reducing the energy spent by the sensing subsystem. Sleep Cycle management are widely used to consider which nodes to put to sleep and the needed duration.

The concept of clustering greatly improves the performance and operation of WSNs. Clustering algorithms conserve energy by grouping nodes and ensuring that a subset of the deployed nodes sends data to the BS. Different clustering algorithms have different underlying concepts for extraction of clusters. The different clustering methodologies may be classified into signal based, distance based and neighbor based. Signal based clustering algorithms form clusters and determines cluster heads based on signal strength. Distance based clustering use distance metrics in making decision and neighbor based clustering considers the neighbor list of each node for cluster formation.

\section{DisTANCE BASED CONCEPTS AND EECS}

Clustering algorithms categorized as distance-based clustering considers the distance metrics when taking decision about routing data. The Clustering Protocol (CP) and Energy Efficient Clustering Scheme (EECS) are common distance based clustering algorithms [12]. The CP algorithm ensures maximum area coverage of the network by forming hexagonal clusters instead of circular ones. The EECS algorithm, which is derived from LEACH, is widely used and bears some common characteristics to LEACH [12]. 
It uses the $\mathrm{CH}$ role rotation of LEACH. EECS improves upon the LEACH algorithm by adopting a weighted cost function. The motivation behind EECS is to overcome the problem of unequal energy dissipation of nodes as a result of their distance by considering energy when electing $\mathrm{CH}$ and using distance as a core factor in building clusters.

EECS solves the problem of unbalanced load and cluster size by using the weighted cost concept. The cost solution ensures that a particular node, $N_{j}$ does not only save its own energy but also save the energy of the $\mathrm{CH}_{i}$ it would join by choosing the $\mathrm{CH}_{i}$ having minimum cost based on the formula:

$$
\operatorname{cost}(j, i)=\left(1-w\left(N_{j}\right)\right) f\left(N_{j}, C H_{i}\right)+w\left(N_{j}\right) g\left(C H_{i}\right)
$$

The effect of applying the distance-based cost is illustrated in Fig. 2. Node $j$ uses the cost function to calculate the cost of the set of $\mathrm{CHs}\left\{\mathrm{CH}_{1}, \mathrm{CH}_{2}, \mathrm{CH}_{3}\right\}$. Though $\mathrm{CH}_{2}$ is closer to $j$, the node selects $\mathrm{CH}_{1}$ as $\mathrm{CH}$ since $\mathrm{CH}_{1}$ has minimum cost.

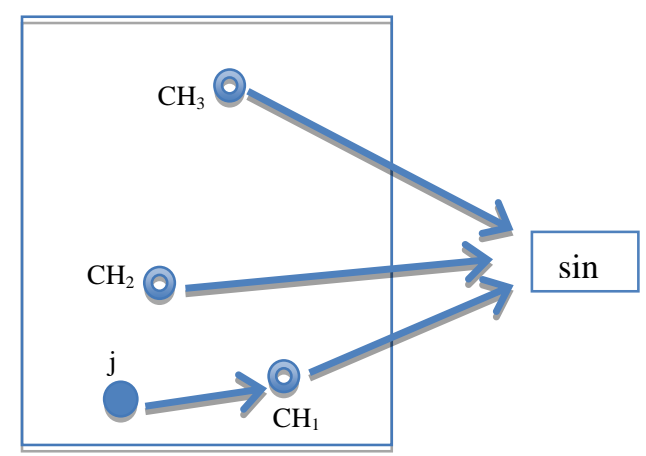

Fig. 2. Plain node considering $\mathrm{CH}$ cost in EECS.

Equation (7) uses normalized functions $f$ and $g$ to represent distance $d\left(P_{j}, C H_{i}\right)$ and $d\left(C_{i}, B S\right)$ respectively as follows:

$$
\begin{gathered}
f\left(P_{j}, C H_{i}\right)=\frac{d\left(P_{j}, C H_{i}\right)}{d_{f-\max }}, \\
g\left(C H_{i}\right)=\frac{d\left(C H_{i}, B S\right)-d_{g-\min }}{d_{g-\max }-d_{g-\min }},
\end{gathered}
$$

where,

$$
\begin{gathered}
d_{f-\max }=\exp \left(\max \left\{d\left(P_{j}, C H_{i}\right)\right\}\right), \\
d_{g-\max }=\max \left\{d\left(C H_{i}, B S\right)\right\}
\end{gathered}
$$

and

$$
d_{g-\min }=\min \left\{d\left(\mathrm{CH}_{i}, \mathrm{BS}\right)\right\} .
$$

The function $w$ is defined as follows:

$$
w\left(P_{j}\right)=c+(1-c) \sqrt{\frac{d\left(P_{j}, B S\right)}{d_{g-\max }-d_{g-\min }}}
$$

Equation 5 ensures that plain nodes choose the nearest cluster head thus reducing intra-cluster communication cost, while (6) guarantees that nodes join the $\mathrm{CH}$ nearby $d\left(\mathrm{CH}_{i}\right.$, $B S$ ) is small thus reducing loads of $\mathrm{CH}$ which are far away from the sink. (7) is the weight off factor for the tradeoff between (5) and (6). The value of $c$ in (7) depends on specific network parameters.

\section{AdAPting the Distance BAsed ConcePt of EECS to SEP}

Studies show that EECS has improved upon homogeneous networks in terms of the stability period [13]. We believe that if the distance based clustering concept is applied to a heterogeneous network considerate improvement will be observed. Since both EECS and SEP are based on LEACH, SEP is chosen as the heterogeneous algorithm. The original cluster head selection phase of SEP is maintained and the distance-based concept of EECS is used for the cluster formation phase.

\section{PERFormance Evaluation}

We consider using the distance based clustering concepts of EECS, which improves over LEACH in a homogeneous network to improve an algorithm in a heterogeneous network. The SEP algorithm is considered since it is derived from LEACH. The cluster head formation is kept same as in the original SEP, and the cluster head selection is done using the weighted cost function, which helps balance the load amongst the cluster heads.

We evaluate the performance of the modified SEP by varying the heterogeneous parameters $(\alpha, \mathrm{m})$ and investigating the improvement of the network lifetime with each of the variations. We simulate the modified SEP under the following different scenarios and analyse its performance compared to the original SEP.

Experiment 1: Large scale heterogeneous network

Experiment 2: Large scale heterogeneous network with extra energy

Experiment 3: Small scale heterogeneous network

Experiment 4: Small scale heterogeneous network with extra energy

We assume the simple radio energy dissipation model considered in SEP, whereby the transmitter dissipates energy to run the radio electronics and the power amplifier, and where the receiver dissipates energy to run the radio electronics. The free space and the multi path fading channel models are used for varying distance between the transmitter and the receiver. If the distance is less than a given threshold, the free space model is used; otherwise, the multi path model is considered. In the different experiments, the common parameters considered are as per table below [7].

\begin{tabular}{|l|l|}
\multicolumn{1}{|c|}{ TABLE I: THE PARAMETER AND VALUE } \\
\hline Area & $100 * 100$ \\
\hline Sink location & $(50,50)$ \\
\hline Init. Energy & $0.5 \mathrm{~J}$ \\
\hline E $_{\text {elec }}$ & $50 \mathrm{~nJ} / \mathrm{Bit}$ \\
\hline Efs & $10 \mathrm{pJ} / \mathrm{bit}^{\prime} / \mathrm{m}^{2}$ \\
\hline Emp & $0.0013 \mathrm{pJ} / \mathrm{bit}^{2} \mathrm{~m}^{4}$ \\
\hline dcrossover & $87 \mathrm{~m}$ \\
\hline E & $5 \mathrm{~nJ} / \mathrm{bit} / \mathrm{signal}$ \\
\hline Packet size & $4000 \mathrm{bits}$ \\
\hline
\end{tabular}

\section{A. Experiment 1 - Parameters and Result}

\begin{tabular}{|l|c|c|c|c|c|}
\hline Parameter & $n$ & $p$ & $m$ & $\alpha$ & $C$ \\
\hline Value & 400 & 0.05 & 0.1 & 1 & 0.6 \\
\hline
\end{tabular}




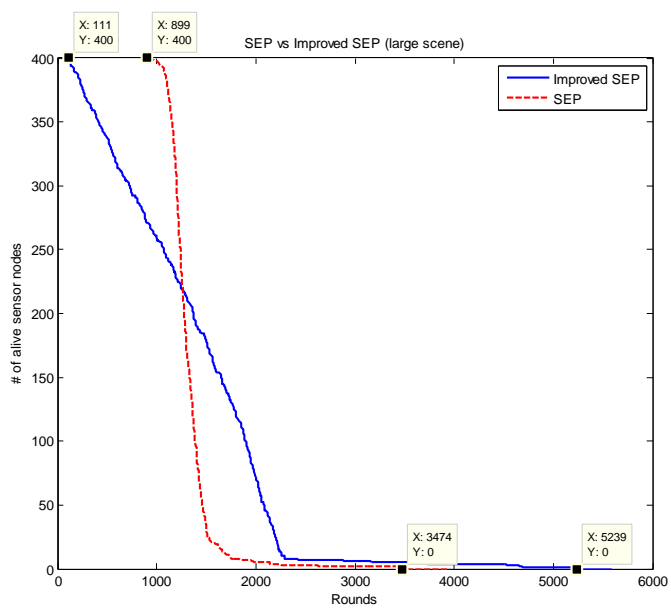

Fig. 3. Experiment 1 - network lifetime.

The simulation result for the first experiment shows that the network lifetime has been prolonged by $50.8 \%$.

\section{B. Experiment 2 - Parameters and Result}

\begin{tabular}{|l|c|c|c|c|c|}
\hline Parameter & $N$ & $p$ & $m$ & $\alpha$ & $c$ \\
\hline Value & 400 & 0.05 & 0.2 & 3 & 0.6 \\
\hline
\end{tabular}

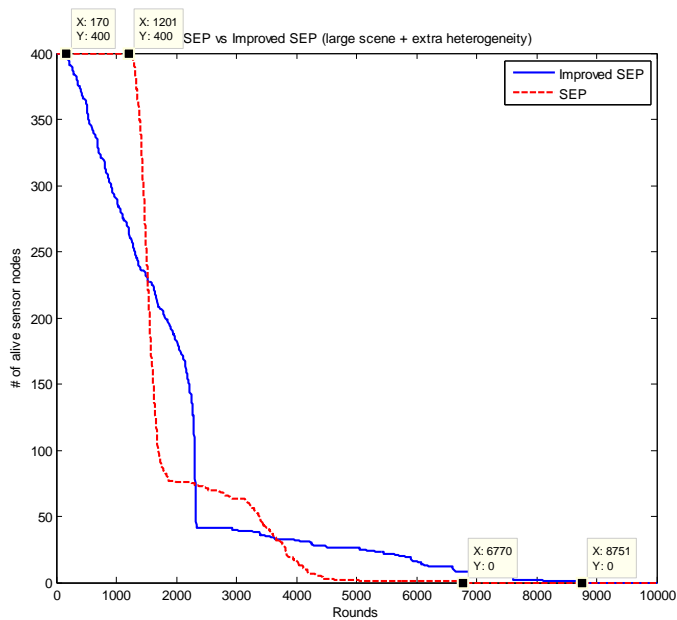

Fig. 4. Experiment 2 - network lifetime.

As shown in Fig. 4, in experiment 2 the network lifetime has been prolonged by $29.26 \%$.

C. Experiment 3 - Parameters and Result

\begin{tabular}{|l|c|c|c|c|c|}
\hline Parameter & $n$ & $p$ & $m$ & $\alpha$ & $c$ \\
\hline Value & 40 & 0.175 & 0.1 & 1 & 0.8 \\
\hline
\end{tabular}

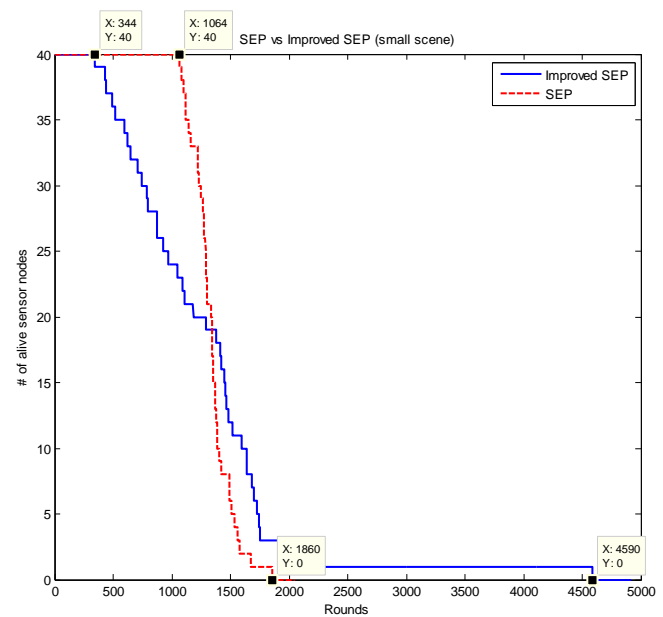

Fig. 5. Experiment 3-network lifetime.
In the third experiment, the network lifetime has been prolonged by $146.77 \%$ as shown in Fig. 5 .

D. Scenario 4 - Parameters and Result

\begin{tabular}{|l|l|l|l|l|l|}
\hline Parameter & $N$ & $P$ & $m$ & $\alpha$ & $C$ \\
\hline Value & 40 & 0.175 & 0.2 & 3 & 0.6 \\
\hline
\end{tabular}

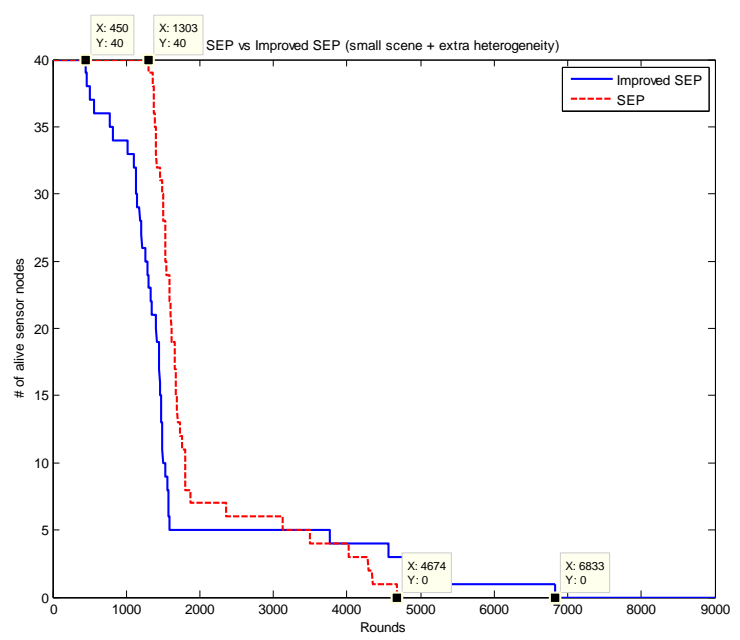

Fig. 6. Experiment 4 - Network lifetime.

The simulation result of the fourth experiment shows that the network lifetime has been prolonged by $46.2 \%$.

\section{CONCLUSION}

In this paper, we have improved on a model heterogeneous network by making it more energy efficient. We have also shown that the modified SEP improves over the original SEP mainly in prolonging the network lifetime. Since longer network lifetime essentially implies longer period of time over which data may be gathered from the network, we conclude that the improved SEP provides better throughput than the original SEP. However, we have noticed that the stability period before the first node death is not improved. As future work, we propose to improve the stability period by implementing the localized competition used in EECS.

\section{REFERENCES}

[1] W. R. Heinzelman, A. Chandrakasan, and H. Balakrishnan, "An application-specific protocol architecture for wireless microsensor networks," IEEE Transactions on Wireless Communications, vol. 1, no. 4, pp. 660-670, 2002.

[2] S. Bandyopadhyay and E. Coyle, "An energy efficient hierarchical clustering algorithm for wireless sensor networks," in Proc. IEEE INFOCOM, vol. 3, pp. 1713-1723, 2003.

[3] C. Intanagonwiwat, R. Govindan, D. Estrin, J. Heidemann, and F. Silva, "Directed diffusion for wireless sensor networking," ACM/IEEE Transactions on Networking, vol. 11, no. 1, pp. 2-16, 2002.

[4] S. Lindsey and C. Raghavendra, "PEGASIS: Power efficient gathering in sensor information systems," in Proc. IEEE Aerospace Conference, Big Sky, USA, 2002, pp. 1125-1130.

[5] I. F. Akyildiz, W. Su, Y. Sankarasubramaniam, and E. Cayirci, "A survey on sensor networks," Communications Magazine IEEE, vol. 40, no. 8, pp. 102-114, August 2002.

[6] G. Smaragdakis, I. Matta, and A. Bestavros, "SEP: A stable election protocol for clustered heterogeneous wireless sensor networks," in. Proc. $2^{\text {nd }}$ International Workshop on Sensor and Actuator Network Protocols and Applications, Boston, USA, 2004.

[7] W. R. Heinzelman, A. Chandrakasan, and H. Balakrishnan, "EnergyEfficient communication protocol for wireless microsensor networks," in Proc. $33^{\text {rd }}$ Hawaii International Conference on System Sciences, USA, 2000. 
[8] J.-H. Chang and L. Tassiulas, "Energy conserving routing in wireless Ad-Hoc networks," in Proc. $19^{\text {th }}$ Annual Joint Conference of the IEEE Computer and Communication Societies, Tel Aviv, 2000, vol. 1, pp. 22-31.

[9] A. Michail and A. Ephremides, "Energy efficient routing for connection-oriented traffic in Ad-Hoc wireless networks," in Proc. $11^{\text {th }}$ IEEE International Symposium on Indoor and Mobile Radio Communications, 2000, vol. 2, pp. 762-766.

[10] C. Intanagonwiwat, R. Govidan, and D. Estrin, "Directed diffusion: A scalable and robust communication paradigm for sensor networks," in Proc. $6^{\text {th }}$ Annual International Conference on Mobile Computing and Networking, USA, 2000, pp. 56-67.

[11] S. S. Saini, R. S. Singh, and V. K. Gupta, "Analysis of energy efficient routing protocols in wireless sensor networks," International Journal of Computer Science and Communications, vol. 1, no. 1, pp. 113-118, 2010.

[12] M. Ye, C. Li, G. Chen, and J. Wu, "EECS: An energy efficient clustering scheme in wireless sensor networks," in Proc. $24^{\text {th }}$ IEEE International Performance, Computing, and Communications Conference, 2005, pp. 535-540.
[13] X. Liu, "A survey on clustering routing protocols in wireless sensor networks," Sensors, vol. 12, no. 8, pp. 11113-111153, 2012.

Nassirah Laloo received the BEng (Hons) degree in computer science and engineering from the University of Mauritius in 2003. She obtained a master's degree with distinction in computer science and engineering in 2006. She is currently working as a lecturer at the University of Technology, Mauritius. She is from the Department of Industrial Systems Engineering at the School of Innovative Technologies and Engineering.

Muhammad Zoubeir Ali Akbar Aungnoo received the BSc (Hons) degree in computer science from the University of Technology, Mauritius in 2011. He obtained a master's degree with distinction in Computer Science from the University of Birmingham, UK.

Mohammad Sameer Sunhaloo received the BSc (Hons) degree in mathematics from the University of Mauritius in 1999. He obtained his PhD in computational mathematics in 2006. He is currently the head of the School of Innovative Technologies and Engineering at the University of Technology Mauritius. 